\title{
Effect of titanium particles on osteoclast activity in vitro
}

\author{
BO MENG ${ }^{1,2}$, XIAOYU YANG ${ }^{2}$, YUE CHEN $^{1}$, JUNJIANG ZHAI ${ }^{1}$ and XING LIANG $^{1}$ \\ ${ }^{1}$ State Key Laboratory of Oral Diseases, Westchina College of Stomatology, Sichuan University, Chengdu 610041; \\ ${ }^{2}$ Guangdong Provincial Stomatological Hospital, Affiliated Stomatological Hospital of the \\ Southern Medical University, Guangzhou 510280, P.R. China
}

Received August 2, 2010; Accepted September 6, 2010

DOI: $10.3892 / \mathrm{mmr} .2010 .368$

\begin{abstract}
Titanium particles generated from orthopedic and dental implants are suspected to play an important role in peri-implant osteolysis. Elucidation of the mechanisms involved in the effects of titanium particles on osteolysis may provide methods for preventing osteolysis. Therefore, in the present study, we investigated the effect of titanium particles on osteoclast activity in vitro. We evaluated bone resorption pits on dentin slices and osteoclast F-actin rings after treatment with titanium particles. The influence of titanium particles on the expression of TRAP, Cat K and CA II mRNA was also evaluated. We found that osteoclast bone resorption activity was enhanced at a lower concentration of titanium particles, but inhibited by a higher concentration of titanium particles. Titanium particles can be phagocytosed by osteoclasts, but the F-actin ring was not destroyed after phagocytosis. In addition, a lower concentration of titanium particles enhanced the mRNA expression of TRAP and Cat $\mathrm{K}$, while higher concentrations of titanium particles decreased their expression. By contrast, the mRNA expression of CA II was decreased at all concentrations of titanium particles. These results suggest that direct exposure to titanium particles at a low concentration enhances osteoclast activity, while exposure to a high concentration inhibits their activity. This understanding of the direct effect of titanium particles on osteoclast activity suggests that aseptic loosening is caused not only by the generation of osteoclasts, but also by enhanced osteoclast activity.
\end{abstract}

\section{Introduction}

Orthopedic and dental implants have been widely used in the clinical setting due to their benefits. However, the longevity of

Correspondence to: Professor Xing Liang, State Key Laboratory of Oral Diseases, Department of Prosthodontics, Westchina Hospital of Stomatology, Sichuan University, Chengdu 610041, P.R. China

E-mail: xingliangdent@gmail.com

Key words: aseptic loosening, titanium particles, osteoclasts, bone resorption an implant is relatively short and implant failure often occurs (1). Aseptic loosening is considered to be the major cause of implant failure, and presents as osteolysis at the bone-implant surface (2,3). Research has demonstrated that titanium particles generated from the titanium implant surface are the main reason for aseptic loosening (4-7), and that the diameter of most of the particles is less than $1 \mu \mathrm{m}(8,9)$.

Several studies have provided evidence supporting the role of titanium particles in the process of bone formation around orthopedic implants. Titanium particles inhibit osteoblast activity, suppress their differentiation and induce apoptosis (10-12). Titanium particles were also found to inhibit the differentiation and activity of bone marrow stem cells $(13,14)$. The mechanism of these effects may involve cytokines released from the cells or phagocytosis of the particles. As a result, titanium particles have an adverse effect on bone formation around the bone-implant surface.

Titanium particles are in direct contact not only with osteoblasts and bone marrow stem cells, but also with osteoclasts. As osteoclasts are generated in the area surrounding the implants, they may be exposed to titanium particles. Thus, it is important to research the reaction of osteoclasts when treated with titanium particles. Unfortunately, there are few reports on the direct effect of titanium particles on mature osteoclast activity.

The objective of this study was to assess the effect of titanium particles on osteoclast activity in vitro. After osteoclasts were exposed to different concentrations of titanium particles, bone resorption activity and the apoptosis of osteoclasts were assessed. The alteration of F-actin rings in osteoclasts and the phagocytosis of titanium particles by cells were also investigated. In addition, variations in the mRNA expression of tartrate-resistant acid phosphatases (TRAP), cathepsin K (Cat K) and carbonic anhydrase II (CA II) were assessed in order to measure the effect of titanium particles on osteoclast activity.

\section{Materials and methods}

Preparation of titanium particles. Commercially pure titanium particles (Alfa Aesar, Milwaukee, WI, USA) with 93\% of the particles $<20 \mu \mathrm{m}$ in diameter were suspended in deionized water $\left(\mathrm{H}_{2} \mathrm{O}\right)$, vortexed and separated according to the variable sedimentation rates of the variously sized particles 
$(12,15)$. After sedimentation, titanium particles were examined using oil-immersion-lens microscopy (x1000). The size of the titanium particles was measured by scanning electron microscopy (SEM), and most of the particle diameters were $<1 \mu \mathrm{m}$ (mean value $\pm \mathrm{SD}, 0.86 \pm 0.66 \mu \mathrm{m})$ (Fig. 1).

Titanium particles were passivated with a $25 \%$ nitric acid wash at $70^{\circ} \mathrm{C}$ for $1 \mathrm{~h}$, washed three times with sterile phosphate-buffered saline (PBS), and then autoclaved at $130^{\circ} \mathrm{C}$ for $60 \mathrm{~min}$ to minimize endotoxin contamination. In preparation for the cell culture, titanium particles were mixed with culture medium under sterile conditions. The culture medium was ultrasonicated for $30 \mathrm{~min}$ in sealed sterile containers before being added to the cell culture.

Osteoclast culture. Osteoclasts were generated by culturing mouse bone marrow cells with osteoblasts. In brief, osteoblasts were prepared from growing calvarial cells from neonatal ICR mice in $\alpha$-minimum essential medium ( $\alpha$-MEM) (Hyclone, Logan, UT, USA) containing 10\% (v/v) fetal calf serum (FCS, Hyclone), $100 \mathrm{U} / \mathrm{ml}$ penicillin and $100 \mu \mathrm{g} / \mathrm{ml}$ streptomycin. Bone marrow cells were obtained from the tibiae and femurs of 6-week-old ICR mice and suspended in $\alpha$-MEM/10\% FCS. The bone marrow cells and osteoblasts were then co-cultured on collagen-coated culture dishes and incubated for 7 days in the presence of $10^{-8} \mathrm{M}$ of $1 \mathrm{a}, 25-(\mathrm{OH})_{2}$ vitamin $\mathrm{D}_{3}$ (Sigma, St. Louis, MO, USA) and $10^{-6} \mathrm{M}$ prostaglandin $\mathrm{E}_{2}\left(\mathrm{PGE}_{2}\right)$ (Alexis). After culturing for 7 days, cells were treated with $0.2 \%$ collagenase at $37^{\circ} \mathrm{C}$ for $10 \mathrm{~min}$, replated on $60-\mathrm{mm}$ dishes and cultured for an additional day. The dishes were then treated with $0.1 \%$ collagenase for $15 \mathrm{~min}$ at $37^{\circ} \mathrm{C}$ and gently pipetted to remove osteoblasts. The remaining cells were stained for TRAP (Sigma) following the manufacturer's instructions, and many of the adherent cells on the dishes were found to be TRAP-positive osteoclasts.

Assessment of bone resorption activity. Osteoclast bone resorption activity was determined by viewing the resorption pits on dentin slices. Osteoclasts were replated on dentin slices that had been placed in 24-well plates and treated with a concentration of $0.05,0.1,0.2$ or $0.4 \mathrm{wt} \%$ titanium particles. A group without titanium particles served as the control. After $24 \mathrm{~h}$, attached osteoclasts were completely removed from the dentin slices by ultrasonic cleaning, and the resorption pits on the dentin slices were stained with toluidine blue. The stained dentin slices were imaged using an inverted phase contrast microscope, resulting in 5 images per slice. Osteoclast resorption activity was quantified by measuring the area of the blue dyed resorption pits with a digital image analysis system (Image Pro-Plus 6.0).

Scanning electron microscopy. Following incubation with $0.1 \mathrm{wt} \%$ titanium particles for $24 \mathrm{~h}$, the cells were washed with PBS three times and fixed in $2.5 \%$ glutaraldehyde for $30 \mathrm{~min}$. After the cells were rinsed with the buffer for $30 \mathrm{~min}$, they received a post-fixation procedure with $1 \%$ osmium tetroxide. The specimens were then dehydrated through ascending ethanol solutions (50, 70, 80, 90 and 100\%) for 15 min each time, and then subjected to critical point drying. The samples were sputtered with gold before being examined using SEM (Inspect-F, FEI).

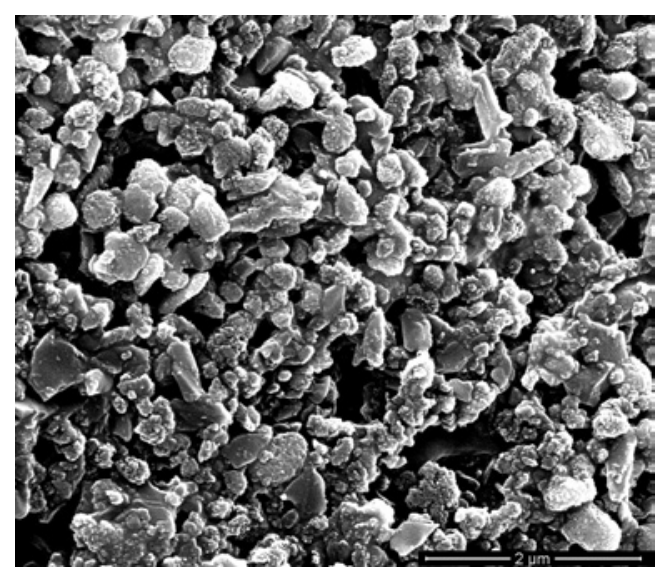

Figure 1. SEM image of the titanium particles used in this study. Most of the particles were $<1 \mu \mathrm{m}$ in diameter.

F-actin staining. After culturing with $0.1 \mathrm{wt} \%$ titanium particles for $24 \mathrm{~h}$, osteoclasts were rinsed with PBS and fixed with $4 \%$ paraformaldehyde for $20 \mathrm{~min}$. The cells were then washed with PBS and further permeabilized with $0.1 \%$ Triton X-100 for $5 \mathrm{~min}$ on ice. Subsequently, the cells were blocked with $1 \%$ bovine serum albumin (BSA) for $1 \mathrm{~h}$ and then stained with phalloidin-TRITC (Millipore) diluted in 1\% BSA for $1 \mathrm{~h}$ in the dark. The cells were subsequently examined for F-actin under a fluorescence inverted phase contrast microscope (Olympus IX71).

Quantitative real-time RT-PCR. The mRNA expression of Cat $\mathrm{K}$, TRAP and CA II was quantified using real-time PCR. After the osteoclasts were treated with titanium particles for $24 \mathrm{~h}$, total RNA was extracted from the cells using TRIzol reagent (Invitrogen). Real-time PCR assays were carried out with $100 \mathrm{ng}$ of purified total RNA using the SensiMix One-Step RT-PCR kit (Quantance, Berlin, Germany) according to the manufacturer's instructions. The primer pairs are listed in Table I. The amplication conditions included denaturation at $95^{\circ} \mathrm{C}$ for $20 \mathrm{sec}$, annealing at $62^{\circ} \mathrm{C}$ for $30 \mathrm{sec}$ and extension at $72^{\circ} \mathrm{C}$ for $30 \mathrm{sec}$ for 40 cycles.

Statistical analysis. Values were averaged and expressed as the means \pm standard deviation (SD). Statistical analysis was performed by analysis of variance (ANOVA), and $\mathrm{p}<0.05$ was considered to be statistically significant.

\section{Results}

Effect of titanium particles on osteoclast bone resorption activity. We investigated whether titanium particles have any effect on osteoclast resorption activity. As shown in Fig. 2, at a low concentration (0.05 wt \%), titanium particles caused a considerable increase in the bone resorption area on the dentin slices, while higher concentrations of titanium particles significantly reduced the area of the resorption pits. This result indicates that titanium particles increased the bone resorption activity of osteoclasts at a low concentration in vitro, while a higher concentration of titanium particles decreased the resorption area. 
Table I. Primer sequences used for real-time PCR analysis.

\begin{tabular}{lll}
\hline Gene & Forward primer sequence & Reverse primer sequence \\
\hline TRAP & CCAGCGACAAGAGGTTCC & TTCCAGCCAGCACATACC \\
Cathepsin K & ATGTGGGTGTTCAAGTTTCTG & ACTGCTTCTGGTGAGTCTTC \\
Carbonic anhydrase II & ACATGGCCTCTTGCTTCTC & GGAAGGGTGAACATTCATCTC \\
$\beta$-actin & AGGTGACAGCATTGCTTCTG & GCTGCCTCAACACCTCAAC \\
\hline
\end{tabular}

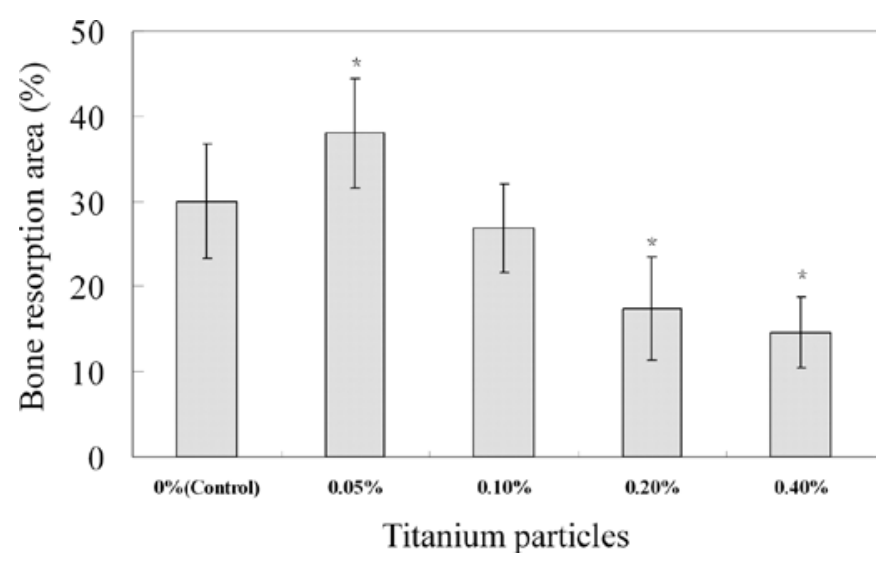

Figure 2. Effect of titanium particles on osteoclast bone resorption activity. The group of $0.05 \mathrm{wt} \%$ titanium particles caused a large area of bone resorption pits, while higher concentrations of titanium particles decreased the resorption area. Osteoclast bone resorption ability was enhanced after treatment with a low concentration of titanium particles. The mean \pm SD of five dentin slice samples from two experiments is shown.

Phagocytosis of titanium particles by osteoclasts. To examine whether osteoclasts can phagocytose titanium particles, we examined cell morphology after treatment with titanium particles by inverted phase contrast microscopy and SEM. As shown in Fig. 3A, nearly all of the particles were within the osteoclasts, and many particles were near the nucleus. Moreover, the pseudopodium of the osteoclasts disappeared (Fig. 3B), indicating the inhibition of osteoclast activity.

Effect of titanium particles on F-actin rings of osteoclasts. We investigated whether titanium particles influence F-actin rings by staining with phalloidin-TRITC. As shown in Fig. 4, in the absence of titanium particles, the F-actin ring was dense and smooth. There was no significant alteration in the osteoclast F-actin ring after the addition of titanium particles. These results suggest that treatment of titanium particles does not disrupt the actin ring, which is necessary for osteoclast bone resorption activity.

Effect of titanium particles on genes related to osteoclast function. We assessed the effect of titanium particles on osteoclast function by analyzing the mRNA expression of Cat K, TRAP and CA II. Semi-quantitative real-time PCR showed that titanium particles at a low concentration increased the mRNA expression of Cat $\mathrm{K}$ and TRAP, while their expression was decreased at a higher concentration of titanium particles (0.05 wt $\%$ ) (Fig. 5A and B). By contrast, the mRNA expres-
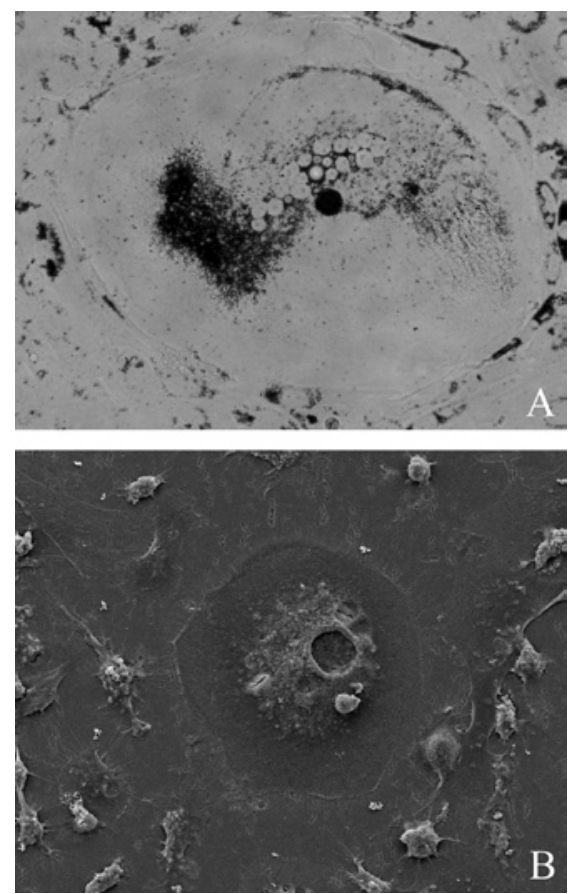

Figure 3. Phagocytosis of titanium particles by osteoclasts. Osteoclasts treated with titanium particles were viewed by light microscopy (A) and SEM (B), respectively. (A) Many particles were found to be located inside the cell after treatment with titanium particles. (B) Pseudopodium disappeared after the phagocytosis of titanium particles by the cells.

sion of CA II was thoroughly suppressed even at a lower concentration of titanium particles (Fig. 5C).

\section{Discussion}

Peri-implant osteolysis is an important concern in the clinical application of both orthopedic and dental implants. Titanium particles generated around the implant surface are considered one of the major causes of osteolysis. Revealing the role of titanium particles in the process of peri-implant osteolysis may provide new methods for preventing osteolysis. In the present study, we evaluated the effect of titanium particles on osteoclast activity in vitro, and found that titanium particles enhanced osteoclast bone resorption activity at a low concentration.

In the present study, titanium particles caused an increase in the area of bone resorption pits on dentin slices at a low concentration $(0.05 \mathrm{wt} \%)$, suggesting that a low concentration of titanium particles promotes osteoclast bone resorption ability in vitro. Upon treating cells with titanium particles at 

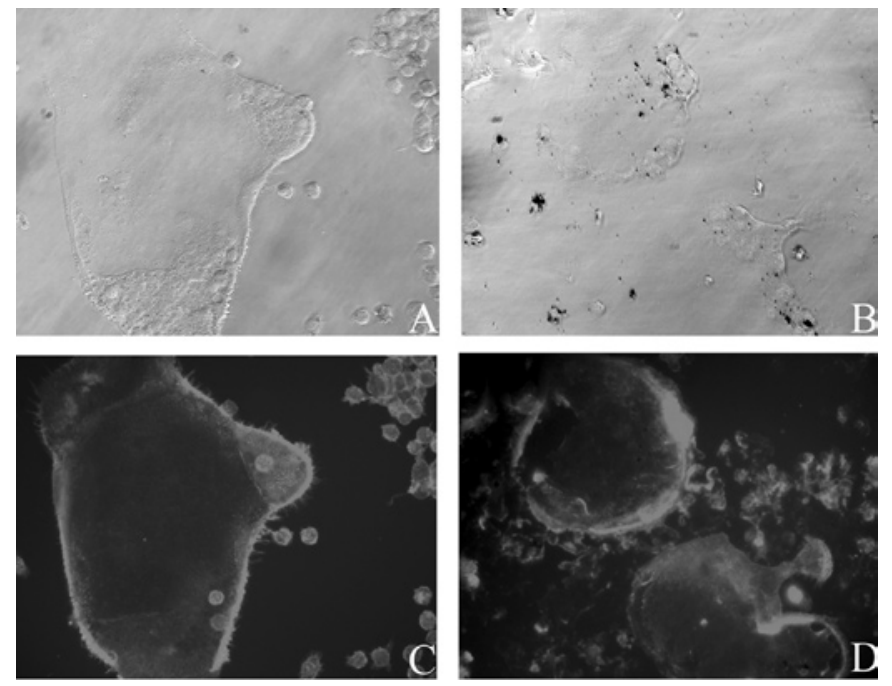

Figure 4. Effect of titanium particles on an osteoclast F-actin ring. After treatment with titanium particles for $24 \mathrm{~h}$, cells were stained with phalloidinTRITC and visualized under visible (A and B) and fluorescent microscopy $(\mathrm{C}$ and $\mathrm{D})$. The actin ring was dense and smooth both in the absence and presence of titanium particles. There was no significant change after treatment with titanium particles (D). Titanium particles were clearly noted within the cell shape, as evidence of phagocytosis.

the beginning of osteoclast generation, researchers found that titanium particles enhanced osteoclast bone resorption ability $(16,17)$. However, this finding contradicts the results of a study by Nakano et al, who found that titanium particles had no effect on osteoclast bone resorption activity or on osteoclast survival (18). This contradiction may be due to the differences between the methods used in the two studies; that is, the culture media contained no cytokines while our media contained $1,25(\mathrm{OH})_{2} \mathrm{D}_{3}$ and $\mathrm{PGE}_{2}$. A research study on the titanium ion, another biocorrosion product of titanium, reported that $\mathrm{Ti}^{4+}$ had an inhibitory effect on the osteoclast activity of in vitrogenerated osteoclasts (19). In addition, Nichols et al showed a decrease in the resorption ability of rat bone-marrow-derived osteoclasts upon exposure to titanium ions (20).

When osteoclasts initiate bone resorption, they undergo active cytoskeleton reorganization to generate an exclusive resorption compartment by forming a circumferential sealing zone. At the sealing zone, the F-actin filament forms an attachment ring structure (21). Consequently, in osteoclasts actively involved in bone resorption, a compact ring structure was usually observed when F-actin was stained. We found that titanium particles had no significant influence on the actin rings in the osteoclasts, indicating that titanium particles do not alter the structure of osteoclasts. Therefore, the effect of titanium particles on osteoclasts was through other pathways and not through the destruction of the F-actin ring.

The effect of titanium particles on osteoclast activity may be due to the phagocytosis of titanium particles (Fig. 3). Many cells, such as osteoblasts, fibroblasts and macrophages, are able to phagocytose titanium particles, and their bioactivity was altered after phaogcytosis $(10,11,22)$. Particles of $1-10 \mu \mathrm{m}$ in diameter can probably be phagocytosed by macrophages and stimulated osteoclastogenesis $(10,22,23)$. In this study, most titanium particles were $<1 \mu \mathrm{m}$ in diameter and all were located inside the cells near the nucleus after co-culturing
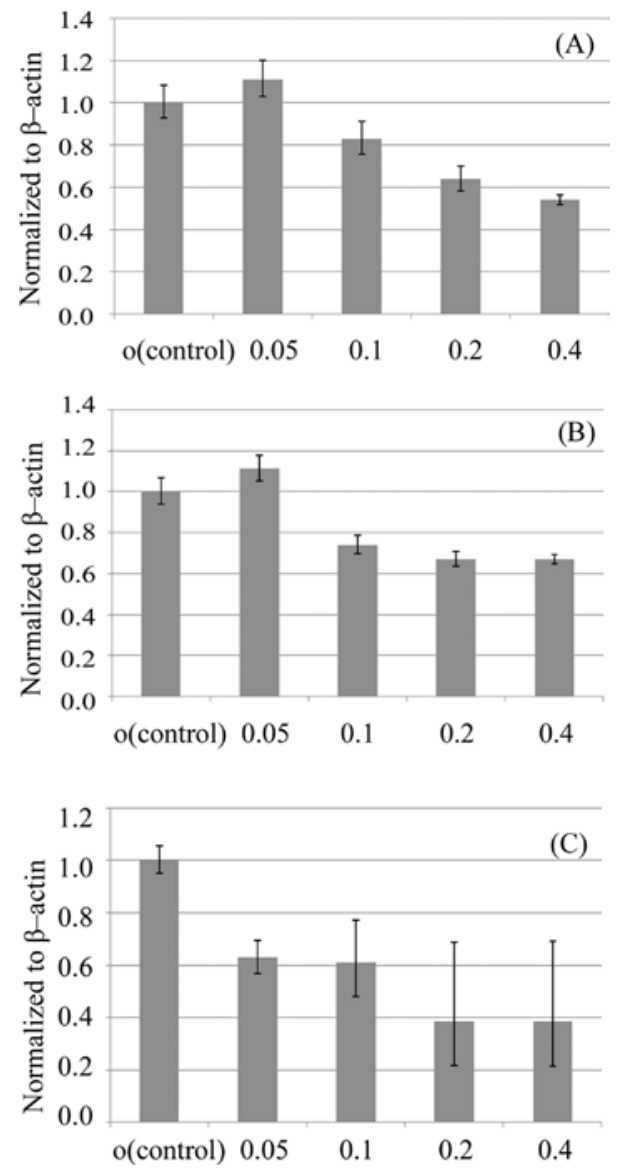

Figure 5. Real-time PCR analysis of the expression of osteoclast markers: (A) TRAP, (B) Cat K and (C) CA II. Results were standardized using $\beta$-actin as a housekeeping gene. Expression of Cat $\mathrm{K}$ and TRAP was increased after treatment with a low concentration of titanium particles, while higher concentrations of titanium particles inhibited their expression. Expression of CA II mRNA was decreased at all concentrations of titanium particles.

with titanium particles for $24 \mathrm{~h}$, indicating the phagocytosis of titanium particles by osteoclasts. Since many particles were near the nucleus, they are likely to affect the expression of genes and proteins. Moreover, after the phagocytosis of titanium particles, the osteoclasts exhibited altered morphology with the disappearance of the pseudopodium. As the pseudopodium is the mark of activated osteoclasts; the disappearance of the microvillus suggested a decrease in osteoclast ability.

TRAP contributes to the intracellular processing of primary bone matrix degradation products and is finally released through the basolateral membrane of osteoclasts. TRAP has been used as a marker of osteoclast function for more than 20 years, and has been shown to be a specific and sensitive indicator of bone resorption $(24,25)$. In the present study, titanium particles dose-dependently decreased TRAP expression, indicating the inhibition of osteoclast activity. Osteoclast bone resorption is caused mainly by acid and lysosomal proteases, which are released through the ruffled border area into the space between the cell membrane and extracellular bone matrix. Cat $\mathrm{K}$, a lysosomal protease, is highly expressed in osteoclasts to degrade the organic matrix (26-28). In this study, titanium particles at a lower concentration slightly enhanced the mRNA expression of Cat K, which 
was probably due to the reaction of osteoclasts after the phagocytosis of titanium particles. However, as the concentration of titanium particles increased, the Cat K mRNA level was suppressed. The data revealed that, in the presence of a low concentration of titanium particles, the Cat $\mathrm{K}$ level in cultured osteoclasts was significantly increased. CA II, an enzyme that degrades the inorganic elements in the bone, is also critical for osteoclast function. We found that titanium particles inhibited the expression of CA II mRNA in the osteoclasts. These findings support the results observed for osteoclast bone resorption ability.

Peri-implant osteolysis is a significant factor involved in the aseptic loosening of dental and orthopedic implants. The net bone loss at the implant-bone interface occurs due to the inhibition of bone formation or the enhancement of bone resorption. Many studies have revealed that titanium particles inhibit bone formation by suppressing osteoblast differentiation or activity. Many investigators have reported that titanium particles promote osteoclast differentiation $(7,16,29)$. In the present study, we found that titanium had a promotive effect on osteoclast activity at a lower concentration. As a result, titanium particles stimulated osteoclast activity in vitro when osteoclasts were in direct contact with a low concentration of titanium particles. Although high concentrations of titanium particles inhibited osteoclast activity, this may still induce bone loss around the implant by enhancing osteoclastogenesis.

In conclusion, our results demonstrated that mature osteoclast activity was enhanced after exposure to a low concentration of titanium particles. In addition, titanium particles can be phagocytosed by osteoclasts, while having no influence on the osteoclast F-actin ring. This understanding of the cellular mechanisms involved in aseptic loosening suggests that aseptic loosening is not caused solely by the generation of osteoclasts, but also by enhanced osteoclast activity.

\section{Acknowledgements}

This research was supported by grants from the National Natural Science Foundation of China (NSFC) (project no. 30672345).

\section{References}

1. Takagi M, Santavirta S, Ida H, et al: High-turnover periprosthetic bone remodeling and immature bone formation around loose cemented total hip joints. J Bone Miner Res 16: 79-88, 2001.

2. Beraudi A, Stea S, Cremonini S, Visentin $M$ and Toni A: Assessment of five interleukins in human synovial fluid as possible markers for aseptic loosening of hip arthroplasty. Artif Organs 33: 538-543, 2009.

3. Andersson MK, Lundberg P, Ohlin A, et al: Effects on osteoclast and osteoblast activities in cultured mouse calvarial bones by synovial fluids from patients with a loose joint prosthesis and from osteoarthritis patients. Arthritis Res Ther 9: R18, 2007.

4. Dorr LD, Bloebaum R, Emmanual J and Meldrum R: Histologic, biochemical, and ion analysis of tissue and fluids retrieved during total hip arthroplasty. Clin Orthop Relat Res 261: 82-95, 1990.

5. Salvati EA, Betts F and Doty SB: Particulate metallic debris in cemented total hip arthroplasty. Clin Orthop Relat Res 293: 160-173, 1993.

6. Wang ML, Sharkey PF and Tuan RS: Particle bioreactivity and wear-mediated osteolysis. J Arthroplasty 19: 1028-1038, 2004.
7. Bauer TW: Particles and periimplant bone resorption. Clin Orthop Relat Res 405: 138-143, 2002.

8. Martini D, Fini M, Franchi M, et al: Detachment of titanium and fluorohydroxyapatite particles in unloaded endosseous implants. Biomaterials 24: 1309-1316, 2003.

9. Franchi M, Orsini E, Martini D, et al: Destination of titanium particles detached from titanium plasma sprayed implants. Micron 38: 618-625, 2007.

10. Pioletti DP, Takei H, Kwon SY, Wood D and Sung KL: The cytotoxic effect of titanium particles phagocytosed by osteoblasts. J Biomed Mater Res 46: 399-407, 1999.

11. Lohmann CH, Schwartz Z, Koster G, et al: Phagocytosis of wear debris by osteoblasts affects differentiation and local factor production in a manner dependent on particle composition. Biomaterials 21: 551-561, 2000.

12. Choi MG, Koh HS, Kluess D, et al: Effects of titanium particle size on osteoblast functions in vitro and in vivo. Proc Natl Acad Sci USA 102: 4578-4583, 2005.

13. Wang ML, Tuli R, Manner PA, Sharkey PF, Hall DJ and Tuan RS: Direct and indirect induction of apoptosis in human mesenchymal stem cells in response to titanium particles. J Orthop Res 21: 697-707, 2003.

14. Meng B, Chen J, Guo D, Ye Q and Liang X: The effect of titanium particles on rat bone marrow stem cells in vitro. Toxicol Mech Methods 29: 552-558, 2009.

15. O'Connor DT, Choi MG, Kwon SY and Paul Sung KL: New insight into the mechanism of hip prosthesis loosening: effect of titanium debris size on osteoblast function. J Orthop Res 22: 229-236, 2004

16. Bi Y, Van De Motter RR, Ragab AA, Goldberg VM, Anderson JM and Greenfield EM: Titanium particles stimulate bone resorption by inducing differentiation of murine osteoclasts. J Bone Joint Surg Am 83: 501-508, 2001.

17. MacQuarrie RA, Fang Chen Y, Coles C and Anderson GI: Wearparticle-induced osteoclast osteolysis: the role of particulates and mechanical strain. J Biomed Mater Res B Appl Biomater 69: 104-112, 2004.

18. Nakano M, Tsuboi T, Kato M, Kurita K and Togari A: Inhibitory effect of titanium particles on osteoclast formation generated by treatment of mouse bone marrow cells with $\mathrm{PGE}_{2}$. Oral Dis 9: 77-83, 2003.

19. Cadosch D, Chan E, Gautschi OP, Meagher J, Zellweger R and Filgueira L: Titanium IV ions induced human osteoclast differentiation and enhanced bone resorption in vitro. J Biomed Mater Res A 91: 29-36, 2009.

20. Nichols KG and Puleo DA: Effect of metal ions on the formation and function of osteoclastic cells in vitro. J Biomed Mater Res 35: 265-271, 1997.

21. Vaananen HK, Zhao H, Mulari M and Halleen JM: The cell biology of osteoclast function. J Cell Sci 113: 377-381, 2000.

22. Fujikawa $\mathrm{Y}$, Itonaga $\mathrm{I}$, Kudo $\mathrm{O}$, Hirayama $\mathrm{T}$ and Taira $\mathrm{H}$ : Macrophages that have phagocytosed particles are capable of differentiating into functional osteoclasts. Mod Rheumatol 15: 346-351, 2005.

23. Lee SH, Brennan FR, Jacobs JJ, Urban RM, Ragasa DR and Glant TT: Human monocyte/macrophage response to cobaltchromium corrosion products and titanium particles in patients with total joint replacements. J Orthop Res 15: 40-49, 1997.

24. Kirstein B, Chambers TJ and Fuller K: Secretion of tartrate-resistant acid phosphatase by osteoclasts correlates with resorptive behavior. J Cell Biochem 98: 1085-1094, 2006.

25. Seol JW, Lee HB, Kim NS, Park SY: Tartrate-resistant acid phosphatase as a diagnostic factor for arthritis. Int J Mol Med 24: 57-62, 2009 .

26. Troen BR: The role of cathepsin $\mathrm{K}$ in normal bone resorption. Drug News Perspect 17: 19-28, 2004.

27. Yamada Y, Ito A, Kojima $\mathrm{H}$, et al: Inhibitory effect of $\mathrm{Zn}^{2+}$ in zinc-containing beta-tricalcium phosphate on resorbing activity of mature osteoclasts. J Biomed Mater Res A 84: 344-352, 2008.

28. Avnet S, Lamolinara A, Zini N, et al: Effects of antisense mediated inhibition of cathepsin $\mathrm{K}$ on human osteoclasts obtained from peripheral blood. J Orthop Res 24: 1699-1708, 2006.

29. Sabokbar A, Pandey R, Quinn JM and Athanasou NA: Osteoclastic differentiation by mononuclear phagocytes containing biomaterial particles. Arch Orthop Trauma Surg 117: 136-140, 1998. 